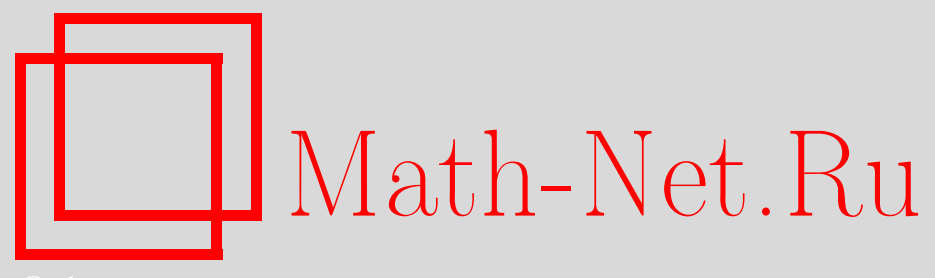

В. Г. Никонов, А. В. Саранцев, О сложности совместной реализации в базисе ДНФ регулярных систем булевых функций, Maтем. вопр. криптогр., 2010, том 1, выпуск 3, 45-65

DOI: https://doi.org/10.4213/mvk15

Использование Общероссийского математического портала Math-Net.Ru подразумевает, что вы прочитали и согласны с пользовательским соглашением http://www.mathnet.ru/rus/agreement

Параметры загрузки:

IP : 52.23 .180 .231

26 апреля 2023 г., $17: 24: 21$ 


\author{
МАТЕМАТИЧЕСКИЕ ВОПРОСЫ КРИПТОГРАФИИ \\ 2010 T. 1 № 3 C. 45-65
}

УДК: 510.52, 512.622

\title{
О сложности совместной реализации в базисе ДНФ регулярных систем булевых функций
}

\author{
В. Г. Никонов ${ }^{1}$, А. В. Саранцев ${ }^{2}$ \\ 1 Академия криптографии Российской Федерации, Москва \\ 2 ООО «Центр сертификационных исследований», Москва
}

Получено 22.IV.2010

Получены оценки сложности совместной реализации регулярных систем булевых функций в базисе элементарных конъюнкций. Показано, что сложность совместной реализации в базисе ортогональных ДНФ всегда равна своему максимальному значению. Описаны функции, порождающие регулярные системы однотипных функций с помощью преобразования из группы Джевонса.

Ключевые слова: сложность реализации, ДНФ, биективные отображения, подстановки, регулярные системы функций, группы преобразований

On the conplexity of joint realization of Boolean functions regular systems in the DNF basis

\author{
V. G. Nikonov ${ }^{1}$, A. V. Sarantsev ${ }^{2}$ \\ 1 Academy of Cryptography of Russian Federation, Moscow \\ 2 LLC "Certification Research Center", Moscow
}

\begin{abstract}
Some complexity estimates of joint realization of Boolean functions regular systems in the DNF basis are derived. It is shown that the complexity of joint realization of such systems in the orthogonal DNF basis is equal to its maximal value. A description of functions whiah may be used to generate regular systems of Boolean functions by means of transforms from the Jevons group is given.

Key words: complexity of realisation, DNF, permutation, regular system of Boolean functions

Citation: Mathematical Aspects of Cryptography, 2010, vol. 1, no. 3, pp. 45-65 (Russian). Работа выполнена при поддержке гранта Президента РФ (НШ-4.2008.10)
\end{abstract}

(C) 2010 В. Г. Никонов, А. В. Саранцев 
В статье рассматривается способ совместной реализации систем функций

$$
f_{1}\left(x_{1}, \ldots, x_{n}\right), \ldots, f_{m}\left(x_{1}, \ldots, x_{n}\right)
$$

в базисе элементарных конъюнкций, при котором конкретная элементарная конъюнкция может участвовать в задании любой из этих функций. Такой способ задает каждую функцию в виде ДНФ и отвечает возможностям современных программируемых логических матриц, при этом общее число $k$ необходимых элементарных конъюнкций является основным сложностным параметром реализации.

В качестве системы (1) в статье анализируются регулярные системы функций

$$
f_{1}\left(x_{1}, \ldots, x_{n}\right), \ldots, f_{n}\left(x_{1}, \ldots, x_{n}\right),
$$

(при этом $m=n$ ), задающие взаимно однозначное отображение $\left(x_{1}, \ldots, x_{n}\right) \mapsto$ $\mapsto\left(y_{1}, \ldots, y_{n}\right)$, где

$$
y_{i}=f_{i}\left(x_{1}, \ldots, x_{n}\right), \quad i \in\{1, \ldots, n\} .
$$

Взаимно однозначные отображения представляют исключительный интерес для криптографических приложений и используются в различных криптографических узлах, поэтому изучению регулярных систем (2) и отображений (3) посвящено большое количество работ $([1-8,13,14])$.

В статье доказывается, что сложность $k$ совместной реализации в базисе ДНФ произвольной регулярной системы булевых функций находится в пределах

$$
n \leq k \leq 2^{n}-1
$$

причем каждое промежуточное значение достижимо.

Наряду с традиционной реализацией в виде ДНФ в статье рассматривается совместная реализация системы функций в базисе ортогональных ДНФ (ОДНФ), когда все элементарные конъюнкции, участвующие в задании функций (1), попарно ортогональны. В настоящее время ОДНФ получили широкое распространение в задачах тестирования, классификации и диагностики, одному из теоретических аспектов задания функций в виде ОДНФ была посвящена статья [12].

В статье доказывается, что сложность $h$ совместной реализации в виде ОДНФ для любой регулярной системы (2) всегда равна своему максимальному значению

$$
h=2^{n}-1 .
$$




\section{1. Геометрический подход к оценке сложности совместной реализации системы булевых функций}

Определение 1. Сложностью совместной реализации, или индексом, системы функций

$$
f_{1}, \ldots, f_{r}
$$

от $n$ переменных $x_{1}, \ldots, x_{n}$ называется минимальное число различных элементарных конъюнкций, из которых может быть составлена ДНФ любой функции $f_{i}$ системы (6).

Заметим, что при $r=1$ индекс системы совпадает с числом конъюнкций кратчайшей ДНФ функции $f_{1}$, или каноническим индексом ([8-11]).

Для нахождения оценок индекса системы функций будем использовать аппарат существенных систем вершин.

Введем необходимые определения, которые для удобства изложения будут иметь преимущественно геометрический характер.

Множество всех векторов $\left(x_{1}, \ldots, x_{n}\right)$ длины $n$ будем называть $n$-мерным единичным кубом $V_{n}$, а векторы $\vec{x}=\left(x_{1}, \ldots, x_{n}\right)$ - его вершинами. Для булевой функции $f\left(x_{1}, \ldots, x_{n}\right)$ определим подмножество $V_{n}$

$$
A_{f}=\left\{\left(x_{1}, \ldots, x_{n}\right) \in V_{n} \mid f\left(x_{1}, \ldots, x_{n}\right)=1\right\},
$$

которое назовем множеством единичных вершин функции. Множество $Q$ единичных вершин элементарной конъюнкции $x_{i_{1}}^{\varepsilon_{i_{1}}} \cdot \ldots \cdot x_{i_{s}}^{\varepsilon_{i_{s}}}$ образует $(n-s)$-мерную грань куба $V_{n}$.

Определение 2. Две вершины $\vec{\alpha}_{1}, \vec{\alpha}_{2} \in A_{f}$ образуют существенную пару вершин в множестве $A_{f}$, если не существует грани $Q$ такой, что $\vec{\alpha}_{1}, \vec{\alpha}_{2} \in$ $Q \subseteq A_{f}$.

Заметим, что две вершины $\vec{\alpha}_{1}, \vec{\alpha}_{2}$ образуют существенную пару тогда и только тогда, когда конъюнкция максимальной длины, принимающая значение 1 на векторах $\vec{\alpha}_{1}, \vec{\alpha}_{2}$, не является импликантой функции $f\left(x_{1}, \ldots, x_{n}\right)$.

Определение 3. Совокупность вершин $\vec{\alpha}_{1}, \ldots, \vec{\alpha}_{t} \in A_{f}$ образует существенную систему в множестве $A_{f}$, если каждая пара $\vec{\alpha}_{i}, \vec{\alpha}_{j}, i \neq j, i, j \in$ $\{1, \ldots, t\}$, является существенной.

Если в множестве $A_{f}$ имеется существенная система вершин мощности $t$, то для канонического индекса $k$ функции $f$ справедлива оценка $k \geq t$. 
Определение 4. Две вершины $\vec{\alpha}_{1}, \vec{\alpha}_{2} \in V_{n}$ назовем существенной парой относительно системы двух функций $f_{1}, f_{2}$, если выполняется хотя бы одно из двух условий:

1) они образуют существенную пару для какой-либо одной из функций $f_{1}, f_{2}$;

2) $\vec{\alpha}_{1}, \vec{\alpha}_{2}$ принадлежат различным множествам $A_{f_{1}}, A_{f_{2}}$ и не существует общей импликанты функций $f_{1}, f_{2}$, принимающей значение 1 на векторах $\vec{\alpha}_{1}, \vec{\alpha}_{2}$.

Отметим, что пара вершин $\vec{\alpha}_{1}=\left(\alpha_{1}^{(1)}, \ldots, \alpha_{n}^{(1)}\right), \vec{\alpha}_{2}=\left(\alpha_{1}^{(2)}, \ldots, \alpha_{n}^{(2)}\right)$ является существенной парой относительно системы функций $f_{1}, f_{2}$ тогда и только тогда, когда вершины $\vec{\alpha}_{1}^{*}=\left(\alpha_{1}^{(1)}, \ldots, \alpha_{n}^{(1)}, \alpha_{n+1}^{(1)}\right), \vec{\alpha}_{2}^{*}=$ $=\left(\alpha_{1}^{(2)}, \ldots, \alpha_{n}^{(2)}, \alpha_{n+1}^{(2)}\right)$ образуют существенную пару для функции

$$
\bar{x}_{n+1} f_{1}\left(x_{1}, \ldots, x_{n}\right) \vee x_{n+1} f_{2}\left(x_{1}, \ldots, x_{n}\right),
$$

где

$$
\begin{array}{ll}
\alpha_{n+1}^{(1)}=0, \text { если } \vec{\alpha}_{1} \in A_{f_{1}}, & \alpha_{n+1}^{(1)}=1, \text { если } \vec{\alpha}_{1} \in A_{f_{2}}, \\
\alpha_{n+1}^{(2)}=0, \text { если } \vec{\alpha}_{2} \in A_{f_{1}}, & \alpha_{n+1}^{(2)}=1, \text { если } \vec{\alpha}_{2} \in A_{f_{2}} .
\end{array}
$$

Пусть задана система функций $f_{1}, \ldots, f_{k}$ и система вершин $\vec{\alpha}_{1}, \ldots, \vec{\alpha}_{t}$, каждая из которых принадлежит ровно одному из множеств $A_{f_{j}}, j \in$ $\in\{1, \ldots, k\}$, положим $\alpha_{i} \in A_{f_{j(i)}}$.

Определение 5. Совокупность вершин $\vec{\alpha}_{1}, \ldots, \vec{\alpha}_{t}$ назовем существенной системой вершин относительно системы функций $f_{1}, \ldots, f_{k}$, если любая пара $\vec{\alpha}_{i_{1}}, \vec{\alpha}_{i_{2}}$ является существенной относительно системы двух функций $f_{j_{1}}, f_{j_{2}}$ таких, что $\vec{\alpha}_{i_{1}} \in A_{f_{j\left(i_{1}\right)}}, \vec{\alpha}_{i_{2}} \in A_{f_{j\left(i_{2}\right)}}$.

Очевидно, что если имеется существенная система вершин мощности $t$ относительно системы функций (6), то для индекса системы (6) справедлива оценка

$$
k \geq t
$$

Существенную систему вершин (ССВ) относительно системы функций $f_{1}, \ldots, f_{r}$ в дальнейшем условимся обозначать через $\Delta\left(f_{1}, \ldots, f_{r}\right)$.

Если построить реализацию системы функций с использованием $k$ элементарных конъюнкций и ССВ мощности $k$, то тем самым будет доказано, что индекс данной системы будет равен $k$. 
Лемма 1. Пусть для системь функиий (6) построена ССВ $\Delta\left(f_{1}, \ldots, f_{r}\right)$, а для вершин $C C B \Delta\left(f_{r+1}\right)$ функиии $f_{r+1}$ выполнено условие

$$
\Delta\left(f_{r+1}\right) \bigcap\left(A_{f_{1}} \cup \ldots \cup A_{f_{r}}\right)=\varnothing .
$$

Тогда объединение двух CCB $\Delta\left(f_{1}, \ldots, f_{r}\right) \cup \Delta\left(f_{r+1}\right)$ образует некоторую ССВ относительно системы функиий $f_{1}, \ldots, f_{r}, f_{r+1}$.

Доказательство леммы следует из того, что вершины, входящие в множество $\Delta\left(f_{r+1}\right)$, не могут быть покрыты общими импликантами совместно с вершинами множества $\Delta\left(f_{1}, \ldots, f_{r}\right)$ в силу условия (8).

\section{2. Оценки изменения сложности совместной реализа- ции регулярной системы функций в базисе ДНФ}

Теорема 1. Индекс $k$ регулярной системы булевых функиий $n$ переменных

$$
f_{1}, \ldots, f_{n}
$$

заключен в пределах

$$
n \leq k \leq 2^{n}-1,
$$

причем для крайних и каждого промежуточного значения $k$ существует регулярная система (9) с таким индексом.

Доказательство. Докажем справедливость оценки индекса $k \geq n$, предположив противное: $k<n$. Пусть $q_{1}, \ldots, q_{k}-$ все различные элементарные конъюнкции, из которых может быть составлена ДНФ любой функции системы (9). Поскольку $k<n$, существует такая функция $f_{j}, 1 \leq j \leq n$, ДНФ которой состоит только из элементарных конъюнкций $q_{i_{1}}, \ldots, q_{i_{m}}$, входящих в ДНФ других функций системы (9). Тогда по законам двойственности произведение функций

$$
\bar{f}_{1} \cdot \ldots \cdot \bar{f}_{j-1} f_{j} \bar{f}_{j+1} \cdot \ldots \cdot \bar{f}_{n}
$$

тождественно равно 0, так как

$$
\bar{f}_{1} \cdot \ldots \cdot \bar{f}_{j-1} f_{j} \bar{f}_{j+1} \cdot \ldots \cdot \bar{f}_{n}=\left(q_{i_{1}} \vee \ldots \vee q_{i_{m}}\right) \cdot \bar{q}_{1} \ldots \bar{q}_{k} .
$$

Последнее противоречит известному критерию Хаффмана, согласно которому для любых $\alpha_{1}, \ldots, \alpha_{n} \in\{0,1\}$

$$
\left\|f_{1}^{\alpha_{1}} \cdot \ldots \cdot f_{n}^{\alpha_{n}}\right\|=1, \quad f_{i}^{0}=\bar{f}_{i}, \quad f_{i}^{1}=f_{i},
$$

и доказывает оценку $k \geq n$. 
Верхняя оценка $k \leq 2^{n}-1$ следует из способа реализации систем функций. Действительно, $2^{n}$ функций вида $\xi_{i}=x_{1}^{\varepsilon_{1}^{(i)}} \ldots x_{n}^{\varepsilon_{n}^{(i)}}$ достаточно для реализации произвольной системы функций, при этом каждая будет представлена в виде совершенной ДНФ. Функции $\xi_{i}$ покрывают все вершины $\left(\varepsilon_{1}^{(i)}, \ldots, \varepsilon_{n}^{(i)}\right)$, $i \in\left\{1, \ldots, 2^{n}\right\}, n$-мерного единичного куба, однако одна из них для регулярной системы является лишней. Это следует из того, что система функций $\bar{f}_{1}, \ldots, \bar{f}_{n}$ также является регулярной и по критерию Хаффмана

$$
\left\|\bar{f}_{1} \cdot \ldots \cdot \bar{f}_{n}\right\|=1
$$

то есть ровно в одной вершине $\left(\varepsilon_{1}^{(j)}, \ldots, \varepsilon_{n}^{(j)}\right)$ все функции системы $f_{1}, \ldots, f_{n}$ принимают значение 0. Соответствующая ей функция $\xi_{i}$ для реализации регулярной системы не требуется, отсюда $k \leq 2^{n}-1$.

Остается доказать, что при любом $k$, заключенном в пределах (10), существует регулярная система (9) с индексом, равным $k$.

Для этого рассмотрим таблицу функций $\left\{f_{k}^{i_{k}}, k \in\{1, \ldots, n\}, i_{k} \in\right.$ $\in\{1, \ldots, k\}\}$, где

$$
\left\{\begin{array}{rll}
f_{1}^{1}=x_{1} ; & & \\
f_{2}^{1}=x_{2}, & f_{2}^{2}=x_{2}+x_{1} ; \\
f_{3}^{1}=x_{3}, & f_{3}^{2}=x_{3}+x_{2}, \quad f_{3}^{3}=x_{3}+x_{2}+x_{1} ; \\
& \ldots & \\
f_{n}^{1}=x_{n}, & f_{n}^{2}=x_{n}+x_{n-1}, \quad \ldots & f_{n}^{n}=x_{n}+\ldots+x_{1} .
\end{array}\right.
$$

Заметим, что любая система функций вида $f_{1}^{i_{1}}, f_{2}^{i_{2}}, \ldots, f_{n}^{i_{n}}$ является треугольной и регулярной по критерию Хаффмана, так как только произведение всех $n$ функций содержит конъюнкцию $x_{1} \cdot x_{2} \cdot \ldots \cdot x_{n}$.

Для каждой функции таблицы $f_{k}^{i_{k}}$ построим CCB $\Delta\left(f_{k}^{i_{k}}\right)$, определяя вершины этой системы удовлетворяющими условиям

$$
\begin{cases}x_{1} & =0, \\ & \cdots \\ x_{k-i_{k}} & =0, \\ f_{k}^{i_{k}} & =x_{k}+\cdots+x_{k-i_{k}+1}=1, \\ x_{k+1} & =1, \\ x_{k+2} & =0 \\ & \cdots \\ x_{n} & =0 .\end{cases}
$$


Любые две различные вершины, для которых выполнены условия (13), отличаются лишь в координатах $x_{k}, \ldots, x_{k-i_{k}+1}$, принадлежат различным компонентам связности линейной функции $f_{k}^{i_{k}}$ и в силу этого действительно образуют ССВ этой функции, причем $\left|\Delta\left(f_{k}^{i_{k}}\right)\right|=2^{i_{k}-1}$. Отметим, что индекс одной функции $f_{k}^{i_{k}}$ в точности равен $2^{i_{k}-1}$, так как $f_{k}^{i_{k}}$ представляется в виде дизъюнкции ровно $2^{i_{k}-1}$ конъюнкций:

$$
f_{k}^{i_{k}}=\bigvee_{\varepsilon_{k}+\ldots+\varepsilon_{k-i_{k}+1}=1} x_{k}^{\varepsilon_{k}} \ldots x_{k-i_{k}+1}^{\varepsilon_{k-i_{k}+1}} .
$$

Рассмотрим теперь произвольную систему функций

$$
f_{1}^{i_{1}}, \ldots, f_{k-1}^{i_{k-1}}, f_{k}^{i_{k}}
$$

и предположим, что для подсистемы $f_{1}^{i_{1}}, \ldots, f_{k-1}^{i_{k-1}}$ построена некоторая ССВ $\Delta\left(f_{1}^{i_{1}}, \ldots, f_{k-1}^{i_{k-1}}\right)$. Покажем, что ССВ для этой системы может быть сформирована из ССВ $\Delta\left(f_{1}^{i_{1}}, \ldots, f_{k-1}^{i_{k-1}}\right)$ и $\Delta\left(f_{k}^{i_{k}}\right)$, построенной по формулам (13), путем их объединения. В самом деле, для вершин $\Delta\left(f_{k}^{i_{k}}\right)$ все координаты $x_{1}, \ldots, x_{k-i_{k}}$ равны 0 , поэтому

$$
\Delta\left(f_{k}^{i_{k}}\right) \bigcap\left(A_{f_{1}^{i_{1}}} \bigcup \ldots \bigcup A_{f_{k-1}^{i_{k-1}}}\right)=\varnothing
$$

и по лемме 1 такое объединение возможно.

Отметим, что добавлению $2^{i_{k}-1}$ вершин $\Delta\left(f_{k}^{i_{k}}\right)$ в ССВ отвечает добавление ровно $2^{i_{k}-1}$ элементарных конъюнкций (14) в реализацию системы функций, откуда следует ее минимальность.

Распространяя процесс объединения ССВ индуктивно на произвольную регулярную систему функций $f_{1}^{i_{1}}, \ldots, f_{n}^{i_{n}}$ таблицы (12), устанавливаем, что

$$
\Delta\left(f_{1}^{i_{1}}, f_{2}^{i_{2}}, \ldots, f_{n}^{i_{n}}\right)=\Delta\left(f_{1}^{i_{1}}\right) \bigcup \Delta\left(f_{2}^{i_{2}}\right) \bigcup \ldots \bigcup \Delta\left(f_{n}^{i_{n}}\right),
$$

причем индекс этой системы функций в точности равен

$$
k=\left|\Delta\left(f_{1}^{i_{1}}\right)\right|+\left|\Delta\left(f_{2}^{i_{2}}\right)\right|+\ldots+\left|\Delta\left(f_{n}^{i_{n}}\right)\right|=2^{0}+2^{i_{2}-1}+\ldots+2^{i_{n}-1}
$$

в силу возможного представления каждой функции системы в виде (14).

Значение $k$, определяемое арифметическим равенством (15), заключено в пределах

$$
n=2^{0}+2^{0}+\ldots+2^{0} \leq k \leq 2^{0}+2^{1}+\ldots+2^{n-1}=2^{n-1}
$$


и при надлежащем выборе индексов $\left(i_{2}, \ldots, i_{n}\right)$ принимает любое промежуточное значение.

Последнее устанавливается методом математической индукции по числу $n$, причем для данного $k$, как нетрудно убедиться, в общем случае существует несколько регулярных систем функций, выбранных из таблицы (12). Например, при $n=4$ и $k=11(4 \leq k \leq 15)$ для числа 11 существует два различных представления (15):

1) $11=2^{0}+2^{1}+2^{2}+2^{2}$;

2) $11=2^{0}+2^{0}+2^{0}+2^{3}$,

которым соответствуют различные регулярные системы с индексом равным 11:
1) $\left\{\begin{array}{l}f_{1}=x_{1} \\ f_{2}=x_{2}+x_{1}, \\ f_{3}=x_{3}+x_{2}+x_{1}, \\ f_{4}=x_{4}+x_{3}+x_{2}\end{array}\right.$
2) $\left\{\begin{array}{l}f_{1}=x_{1}, \\ f_{2}=x_{2}, \\ f_{3}=x_{3}, \\ f_{4}=x_{4}+x_{3}+x_{2}+x_{1} .\end{array}\right.$

\section{3. Сложность совместной реализации регулярной системы функций в базисе ортогональных ДНФ (ОДНФ)}

Напомним, что две элементарные конъюнкции $q_{1}=x_{i_{1}}^{\varepsilon_{i_{1}}} \cdot \ldots \cdot x_{i_{r}}^{\varepsilon_{i_{r}}}$ и $q_{2}=$ $=x_{j_{1}}^{\delta_{j_{1}}} \cdot \ldots \cdot x_{j_{r}}^{\delta_{j_{r}}}$ попарно ортогональны, если при любых значениях переменных их произведение равно нулю:

$$
q_{1} \cdot q_{2} \equiv 0
$$

Известно, что элементарные конъюнкции $q_{1}$ и $q_{2}$ ортогональны тогда и только тогда, когда в задание одной из них некоторая переменная входит в прямом, а в задание другой - в инверсном виде.

Определение 6. Сложностью $h$ совместной реализации в базисе ортогональных ДНФ (ОДНФ), или ортогональным индексом, системы функций

$$
f_{1}, \ldots, f_{r}
$$


от $n$ переменных $x_{1}, \ldots, x_{n}$ назовем минимальное число различных попарно ортогональных элементарных конъюнкций, из которых может быть составлена ДНФ любой функции системы.

При $r=1$ ортогональный индекс системы совпадает с числом элементарных конъюнкций в кратчайшей ОДНФ (см. [12]).

Теорема 2. Ортогональный индекс любой регулярной системы (2) функиий от п переменных равен

$$
h=2^{n}-1 .
$$

Доказательство. Из теоремы 1 и того, что реализация в базисе ОДНФ является частным случаем реализации в виде ДНФ, вытекает, что ортогональный индекс заключен в пределах (10)

$$
n \leq h \leq 2^{n}-1 \text {. }
$$

Предположим теперь, что в представлении регулярной системы (2) в виде ОДНФ найдется элементарная конъюнкция

$$
q=x_{j_{1}}^{\varepsilon_{j_{1}}} \cdot \ldots \cdot x_{j_{l}}^{\varepsilon_{j_{l}}},
$$

причем $l \leq n-1$. Элементарная конъюнкция $q$ задает подкуб $Q n$-мерного единичного куба $V_{n}$, причем $|Q|=2^{n-l} \geq 2$.

Очевидно, элементарная конъюнкция $q$ не может одновременно участвовать в задании всех функций $f_{1}, \ldots, f_{n}$ регулярной системы (2), так как в противном случае

$$
\left\|f_{1} \cdot \ldots \cdot f_{n}\right\| \geq\|q\|=|Q|=2^{n-l} \geq 2,
$$

что противоречит критерию Хаффмана (11), согласно которому при $\alpha_{1}=\ldots=$ $=\alpha_{n}=1$

$$
\left\|f_{1} \cdot \ldots \cdot f_{n}\right\|=1
$$

Пусть элементарная конъюнкция $q$ участвует в задании некоторых функций регулярной системы

$$
f_{i_{1}}, \ldots, f_{i_{s}}
$$

а в задании остальных функций

$$
f_{i_{s+1}}, \ldots, f_{i_{n}}
$$

не участвует, $1<s<n$. 
Для функций (17), очевидно, выполнено условие

$$
q \cdot f_{i_{j}} \equiv q, \quad j \in\{1, \ldots, s\}
$$

так как элементарная конъюнкция $q$ входит в задание каждой из них.

Если же элементарная конъюнкция $q$ не участвует в задании функции $f_{i_{\nu}}$, то

$$
q \cdot f_{i_{\nu}} \equiv 0,
$$

так как все присутствующие в задании функции $f_{i_{\nu}}$ элементарные конъюнкции ортогональны $q$.

Поэтому для всех функций (18) справедливо условие

$$
q \cdot \bar{f}_{i_{\nu}} \equiv q, \quad \nu \in\{s+1, \ldots, n\} .
$$

Из условий (19), (20) вытекает, что

$$
f_{i_{1}} \cdot \ldots \cdot f_{i_{s}} \cdot \bar{f}_{i_{s+1}} \cdot \ldots \cdot \bar{f}_{i_{n}}=q
$$

и

$$
\left\|f_{i_{1}} \cdot \ldots \cdot f_{i_{s}} \cdot \bar{f}_{i_{s+1}} \cdot \ldots \cdot \bar{f}_{i_{n}}\right\|=\|q\|=|Q|=2^{n-l} \geq 2,
$$

что противоречит критерию Хаффмана (11) при $\alpha_{i_{1}}=\ldots=\alpha_{i_{s}}=1$ и $\alpha_{i_{s+1}}=$ $=\ldots=\alpha_{i_{n}}=0$, по которому

$$
\left\|f_{i_{1}} \cdot \ldots \cdot f_{i_{s}} \cdot \bar{f}_{i_{s+1}} \cdot \ldots \cdot \bar{f}_{i_{n}}\right\|=1 .
$$

Тем самым установлено, что в представлении регулярной системы (2) в виде ОДНФ все элементарные конъюнкции имеют максимальную длину, равную $n$. Каждая такая элементарная конъюнкция покрывает лишь одну вершину $n$-мерного единичного куба. Всего же для задания регулярной системы (2), как было отмечено в ходе доказательства теоремы 1, необходимо покрыть ровно $2^{n}-1$ вершин, следовательно,

$$
h=2^{n}-1 .
$$

Теорема 2 доказана. 


\section{4. Реализация регулярной системы с помощью одно- типных функций}

Оценки (10) и (16) показывают высокую возможную сложность совместной реализации регулярных систем (2). В этой связи особый интерес представляют так называемые регулярные системы однотипных функций, когда каждая функция системы (2) получается из одной порождающей функции $f_{0}\left(x_{1}, \ldots, x_{n}\right)$ с помощью некоторого преобразования $\sigma_{i}$ из группы Джевонса, называемого также преобразованием однотипности:

$$
f_{i}\left(x_{1}, \ldots, x_{n}\right)=f_{0}\left(\sigma_{i}\left(x_{1}, \ldots, x_{n}\right)\right) .
$$

Наряду с описанной выше совместной реализацией, когда значения всех функций вырабатываются параллельно, такие системы допускают реализацию в последовательном режиме. Последовательный режим предполагает задание только функции $f_{0}\left(x_{1}, \ldots, x_{n}\right)$, из которой простыми преобразованиями $\sigma_{i}$ генерируются $f_{i}\left(x_{1}, \ldots, x_{n}\right)$. В этом случае важнейшим сложностным параметром оказывается число $t$ элементарных конъюнкций в кратчайшей ДНФ функции $f_{0}\left(x_{1}, \ldots, x_{n}\right)$.

Известно, что при фиксированном значении $t$ число сбалансированных булевых функций, способных породить регулярную систему (21), конечно (см. [12]), и все они могут быть перечислены.

С ростом $t$ усложняется строение порождающей функции $f_{0}\left(x_{1}, \ldots, x_{n}\right)$ и увеличивается количество таких функций. Для некоторых функций $f_{0}\left(x_{1}, \ldots, x_{n}\right)$ координатные функции системы (21) могут существенно зависеть лишь от ограниченного числа переменных, а для отдельных $f_{0}\left(x_{1}, \ldots, x_{n}\right)$ - от всех $n$ переменных. При этом необходимо подчеркнуть, что лишь узкий класс булевых функций может породить регулярную систему (21) однотипных функций.

При $t=1$ функция $f_{0}$ имеет вид $f_{0}=x_{i}$ и порождает регулярную систему при любом $n$, но эта система имеет исключительно простой вид, сводящийся к перестановке переменных

$$
y_{j}=x_{i}, \quad i, j \in\{1, \ldots, n\} .
$$

При $t=2$ порождающая функция определяется однозначно с точностью до класса эквивалентности относительно группы Джевонса:

$$
f_{0}=x_{1} x_{2} \vee \bar{x}_{1} x_{3}
$$


при этом регулярная система зависит лишь от 3 переменных, в частности:

$$
\begin{aligned}
& f_{1}\left(x_{1}, x_{2}, x_{3}\right)=x_{1} x_{2} \vee \bar{x}_{1} x_{3}, \\
& f_{2}\left(x_{1}, x_{2}, x_{3}\right)=\bar{x}_{1} \bar{x}_{2} \vee x_{2} x_{3}, \\
& f_{3}\left(x_{1}, x_{2}, x_{3}\right)=x_{1} x_{2} \vee \bar{x}_{2} x_{3} .
\end{aligned}
$$

Заметим, что уже при $t=3$ существует функция $f_{0}\left(x_{1}, x_{2}, x_{3}\right)=x_{1} x_{2} \vee$ $\vee x_{1} x_{3} \vee x_{2} x_{3}$, порождающая регулярные системы однотипных функций как для $n=3$, например,

$$
\begin{aligned}
& f_{1}\left(x_{1}, x_{2}, x_{3}\right)=x_{1} x_{2} \vee x_{1} x_{3} \vee x_{2} x_{3}, \\
& f_{2}\left(x_{1}, x_{2}, x_{3}\right)=x_{1} x_{2} \vee x_{1} \bar{x}_{3} \vee x_{2} \bar{x}_{3}, \\
& f_{3}\left(x_{1}, x_{2}, x_{3}\right)=\bar{x}_{1} x_{2} \vee \bar{x}_{1} x_{3} \vee x_{2} x_{3},
\end{aligned}
$$

так и для $n=4$ :

$$
\begin{aligned}
& f_{1}\left(x_{1}, x_{2}, x_{3}, x_{4}\right)=x_{1} x_{2} \vee x_{1} x_{3} \vee x_{2} x_{3}, \\
& f_{2}\left(x_{1}, x_{2}, x_{3}, x_{4}\right)=x_{1} \bar{x}_{2} \vee x_{1} x_{4} \vee \bar{x}_{2} x_{4}, \\
& f_{3}\left(x_{1}, x_{2}, x_{3}, x_{4}\right)=x_{1} \bar{x}_{3} \vee x_{1} \bar{x}_{4} \vee \bar{x}_{3} \bar{x}_{4}, \\
& f_{4}\left(x_{1}, x_{2}, x_{3}, x_{4}\right)=x_{2} \bar{x}_{3} \vee x_{2} x_{4} \vee \bar{x}_{3} x_{4} .
\end{aligned}
$$

Причем при бо́льших $n$ эта функция регулярных систем не порождает.

Поиск функций, порождающих регулярные системы от сколь угодно большого числа переменных, является нетривиальной задачей. Поэтому представляет интерес приведение положительного примера ее разрешения уже при $t=3$. Успех был достигнут при построении регулярной системы, в которой переход от одной функции к другой осуществлялся с помощью преобразования $\delta_{l}$, реализуемого аффинным регистром сдвига (см. [14]) с функцией $l\left(x_{1}, \ldots, x_{n}\right)=x_{1}+\sum_{i=2}^{n} b_{i} x_{i}+b_{0}, b_{0}, b_{i} \in\{0,1\}, i \in\{2, \ldots, n\}$, обратной связи.

Теорема 3 ([14]). Пусть $n \geq 5$, f - нелинейная функция, зависящая существенно только от первых трех переменных. Тогда система функиий

$$
\left(f\left(x_{1}, \ldots, x_{n}\right), f\left(\delta_{l}\left(x_{1}, \ldots, x_{n}\right)\right), \ldots, f\left(\delta_{l}^{n-1}\left(x_{1}, \ldots, x_{n}\right)\right)\right)
$$

регулярна в каждом из следующих случаев для нечетного $n$ :

1) $f=x_{2}+x_{3}+x_{1} x_{2}+a \quad$ u $l=x_{1}+b x_{n-1}$,

2) $f=x_{1}+x_{3}+x_{1} x_{2}+a \quad u \quad l=x_{1}+b x_{n-1}+b$,

3) $f=x_{1}+x_{2}+x_{2} x_{3}+a \quad u \quad l=x_{1}+b x_{3}$, 
4) $f=x_{1}+x_{3}+x_{2} x_{3}+a \quad u \quad l=x_{1}+b x_{3}+b$, где $a, b \in\{0,1\}$, «+»-операция сложения по модулю 2.

В частном случае, когда аффинный регистр сводится к циклическому сдвигу (при $b=0)$, теорема свидетельствует о существовании регулярных систем однотипных функций для любого $n \geq 3$, построенных на базе указанных функций 3 переменных. Все эти функции, как нетрудно видеть, однотипны и имеют индекс $t=3$. В частности, функция

$$
f\left(x_{1}, x_{2}, x_{3}\right)=x_{1}+x_{2}+x_{2} x_{3}
$$

имеет кратчайшую ДНФ вида

$$
f\left(x_{1}, x_{2}, x_{3}\right)=x_{1} \bar{x}_{2} \vee x_{1} x_{3} \vee \bar{x}_{1} x_{2} \bar{x}_{3}
$$

Докажем, что существуют функции индекса $t=4$, порождающие регулярные системы однотипных функций от сколь угодно большого числа переменных.

Для удобства изложения результатов будем нумеровать координаты двоичных векторов, начиная с 0 . Пусть далее:

$V_{n}$ - множество двоичных векторов $\boldsymbol{x}=\left(x_{0}, \ldots, x_{n-1}\right)$ размерности $n$;

$f(\boldsymbol{x})=f\left(x_{0}, \ldots, x_{n-1}\right)-$ двоичная функция от $n$ переменных;

$S\left(V_{n}\right)$ - группа подстановок на $V_{n}$;

$\sigma \in S\left(V_{n}\right), \sigma^{-1}:\left(x_{0}, \ldots, x_{n-1}\right) \mapsto\left(x_{1}, \ldots, x_{n-1}, x_{0}\right)$;

$f^{\sigma}=f\left(\boldsymbol{x}^{\sigma^{-1}}\right)$ - действие $\sigma$ на $f$;

$\mathcal{C}_{\pi}=\left(f_{0}, \ldots, f_{n-1}\right)-$ система координатных функций отображения $\pi: V_{n} \rightarrow V_{n}$; $\mathcal{C}(f ; \sigma)=\left(f, f^{\sigma}, \ldots, f^{\sigma^{n-1}}\right)$.

Лемма 2. Пусть $f \in \mathcal{F}_{n}$. Если суперпозиция $h\left(f, f^{\sigma}, \ldots, f^{\sigma^{n-1}}\right)=x_{0}$ для некоторой функичи $h \in \mathcal{F}_{n}$, то системы функиий $\mathcal{C}(f ; \sigma)$ u $\mathcal{C}(h ; \sigma)$ являются регулярными, и подстановки $\alpha, \beta \in S\left(V_{n}\right)$, задаваемые этими системами, являются взаимно обратными, то есть $\alpha=\beta^{-1}$.

Доказательство. Пусть $\alpha$ и $\beta$ - отображения $V_{n} \rightarrow V_{n}$, системы координатных функций которых есть $\mathcal{C}(f ; \sigma)$ и $\mathcal{C}(h ; \sigma)$ соответственно. Отображение $\alpha: \boldsymbol{x} \mapsto \boldsymbol{y}$ имеет вид

$$
y_{i}=f^{\sigma^{i}}(\boldsymbol{x})=f\left(x_{i}, \ldots, x_{n+i-1}\right), \quad i \in\{0, \ldots, n-1\},
$$

где суммирование в индексах переменных проводится по модулю $n$. 
Для координатных функций $h^{\sigma^{i}}, i \in \overline{0, n-1}$, отображения $\beta$ справедливы соотношения:

$h^{\sigma^{i}}(\boldsymbol{y})=h\left(y_{i}, \ldots, y_{n+i-1}\right)=h\left(f\left(x_{i}, \ldots, x_{n+i-1}\right), \ldots, f\left(x_{n+i-1}, \ldots, x_{n+i-2}\right)\right)=x_{i}$, поскольку $h\left(f, f^{\sigma}, \ldots, f^{\sigma^{n-1}}\right)=x_{0}$. Это означает, что $\alpha=\beta^{-1}$.

Пусть $\omega \in S\left(V_{n}\right)$ - такая подстановка, что

$$
\omega: \boldsymbol{x}=\left(x_{0}, x_{1}, \ldots, x_{n-2}, x_{n-1}\right) \mapsto\left(x_{n-1}, x_{n-2}, \ldots, x_{1}, x_{0}\right), \quad \boldsymbol{x} \in V_{n} .
$$

Имеет место равенство

$$
\sigma \cdot \omega=\omega \cdot \sigma^{-1}
$$

Лемма 3. Пусть $\pi \in S\left(V_{n}\right)-$ такая подстановка, что $\mathcal{C}_{\pi}=\mathcal{C}(f ; \sigma)$ и $\mathcal{C}_{\pi^{-1}}=\mathcal{C}(h ; \sigma)$ для некоторых функиий $f, h \in \mathcal{F}_{n}$. Тогда для произвольного $l \in \mathbb{Z}$ системы $\mathcal{C}\left(f^{\sigma^{l} \omega} ; \sigma\right)$ и $\mathcal{C}\left(h^{\sigma^{-(l+2)} \omega} ; \sigma\right)$ регулярны и для подстановок $\alpha, \beta \in S\left(V_{n}\right), \mathcal{C}_{\alpha}=\mathcal{C}\left(f^{\sigma^{l} \omega} ; \sigma\right), \mathcal{C}_{\beta}=\mathcal{C}\left(h^{\sigma^{-(l+2)} \omega} ; \sigma\right)$ верно соотнотение $\alpha^{-1}=\beta$.

Доказательство. Рассмотрим систему координатных функций подстановки $\omega \pi \omega$

$$
\begin{aligned}
\mathcal{C}_{\omega \pi \omega}=\left(f^{\omega}, \ldots, f^{\sigma^{i} \omega}, \ldots, f^{\sigma^{n-1} \omega}\right)^{\omega}= & \left(f^{\omega}, \ldots, f^{\omega \sigma^{-i}}, \ldots, f^{\omega \sigma^{1-n}}\right)^{\omega}= \\
& =\left(f^{\omega \sigma}, f^{\omega \sigma^{2}}, \ldots, f^{\omega \sigma^{n}}\right)=\mathcal{C}\left(f^{\omega \sigma} ; \sigma\right) .
\end{aligned}
$$

Тогда для $l \in \mathbb{Z}$ система координатных функций подстановки $\sigma^{l+1} \omega \pi \omega$ удовлетворяет соотношению

$$
\mathcal{C}_{\sigma^{l+1} \omega \pi \omega}=\mathcal{C}\left(f^{\omega \sigma^{-l}} ; \sigma\right)=\mathcal{C}\left(f^{\sigma^{l} \omega} ; \sigma\right)
$$

Значит, система $\mathcal{C}\left(f^{\sigma^{l} \omega} ; \sigma\right)$ регулярна.

Пусть $\alpha \in S\left(V_{n}\right)$ и $\mathcal{C}_{\alpha}=\mathcal{C}\left(f^{\sigma^{l} \omega} ; \sigma\right)$, тогда $\alpha=\sigma^{l+1} \omega \pi \omega$. Рассмотрим систему координатных функций подстановки $\alpha^{-1}=\omega \pi^{-1} \omega \sigma^{-1-l}$ :

$$
\begin{aligned}
\mathcal{C}_{\alpha^{-1}} & =\left(h^{\omega}, \ldots, h^{\sigma^{i} \omega}, \ldots, h^{\sigma^{n-1} \omega}\right)^{\omega \sigma^{-1-l}}=\left(h^{\sigma^{n-1} \omega}, \ldots, h^{\sigma^{i} \omega}, \ldots, h^{\omega}\right)^{\sigma^{-1-l}}= \\
& =\left(h^{\omega \sigma^{1-n}}, \ldots, h^{\omega \sigma^{-i}}, \ldots, h^{\omega}\right)^{\sigma^{-(1+l)}}=\mathcal{C}\left(h^{\omega \sigma^{l+2}} ; \sigma\right)=\mathcal{C}\left(h^{\sigma^{-(l+2)} \omega} ; \sigma\right) .
\end{aligned}
$$

Значит, система $\mathcal{C}\left(h^{\sigma^{-(l+2)} \omega} ; \sigma\right)$ регулярна и задаваемая ею подстановка $\beta \in S\left(V_{n}\right)$ равна $\alpha^{-1}$. 
Теорема 4. Система $\mathcal{C}(f ; \sigma)$ регулярна в каждом из следующих случаев:

1) $n \geq 5, f=x_{1}+x_{0} \bar{x}_{2} x_{3}$ или $f=x_{2}+x_{0} \bar{x}_{1} x_{3}$;

2) $n \geq 5$, $n$ - нечетное, $f=x_{1}+x_{0} x_{2} \bar{x}_{3}$ или $f=x_{2}+\bar{x}_{0} x_{1} x_{3}$;

3) $n \geq 5$, п не делится на 3,

(a) $f=x_{0}+x_{1} x_{2} \bar{x}_{3}$ или $f=x_{3}+\bar{x}_{0} x_{1} x_{2}$,

(b) $f=x_{0}+\bar{x}_{1} x_{2} x_{3}$ или $f=x_{3}+x_{0} x_{1} \bar{x}_{2}$.

Каждая из указанных функций порождает регулярную систему с помощью циклического сдвига координат только для представленных значений $n$.

Доказательство. Пусть отображение $\alpha: V_{n} \rightarrow V_{n}$ задается системой координатных функций $\mathcal{C}(f ; \sigma), f \in \mathcal{F}_{n}, \alpha: \boldsymbol{x} \mapsto \boldsymbol{y}, y_{i}=f^{\sigma^{i}}(\boldsymbol{x})$. В каждом из случаев укажем функцию $h \in \mathcal{F}_{n}$ и докажем, что она удовлетворяет условию леммы 2.

1) Если $f(\boldsymbol{x})=x_{1}+x_{0} \bar{x}_{2} x_{3}$, то $y_{i}=x_{i+1}+x_{i} \bar{x}_{i+2} x_{i+3}, i \in\{0, \ldots, n-1\}$. Здесь и далее вычисление индексов у переменных производится по модулю $n$. Пусть $h(\boldsymbol{y})=y_{n-1}+\bar{y}_{0} y_{1} y_{n-2}$, тогда

$$
\begin{gathered}
h(\boldsymbol{y})=x_{0}+x_{n-1} \bar{x}_{1} x_{2}+\left[\bar{x}_{1}+x_{0} \bar{x}_{2} x_{3}\right]\left[x_{2}+x_{1} \bar{x}_{3} x_{4}\right]\left[x_{n-1}+x_{n-2} \bar{x}_{0} x_{n+1}\right]= \\
=x_{0}+x_{n-1} \bar{x}_{1} x_{2}+\left[\bar{x}_{1}+x_{0} \bar{x}_{2} x_{3}\right] \times \\
\times\left[x_{n-1} x_{2}+x_{n-2} \bar{x}_{0} x_{1} x_{2}+x_{n-1} x_{1} \bar{x}_{3} x_{4}+x_{n-2} \bar{x}_{0} x_{1} \bar{x}_{3} x_{4}\right]= \\
=x_{0}+x_{n-1} \bar{x}_{1} x_{2}+x_{n-1} \bar{x}_{1} x_{2}=x_{0} .
\end{gathered}
$$

2) Пусть $f\left(x_{0}, \ldots, x_{n-1}\right)=x_{1}+x_{0} x_{2} \bar{x}_{3}$. Если $n$ четно, то группа $\langle\sigma\rangle$ имеет орбиту из двух векторов $(0,1, \ldots, 0,1)$ и $(1,0, \ldots, 1,0)$. Поскольку $f(0,1, \ldots, 0,1)=f(1,0, \ldots, 1,0)=1$, при четных значениях $n$ функция $f$ не порождает регулярной системы $\mathcal{C}(f ; \sigma)$.

Пусть $n \geq 5, n-$ нечетное. В рассматриваемом случае

$$
y_{i}=x_{i+1}+x_{i} x_{i+2} \bar{x}_{i+3}, \quad i \in\{0, \ldots, n-1\}
$$

Выберем функцию $h(\boldsymbol{y})=y_{n-1}+y_{n-2} \cdot\left[\sum_{t=0, t \text {-четно }}^{n-5} y_{0} y_{2} \cdot \ldots \cdot y_{t} \bar{y}_{t+1}\right]$. 
Для четного $t \leq n-5$ рассмотрим слагаемое $y_{0} y_{2} \cdot \ldots \cdot y_{t} \bar{y}_{t+1}$. Заметим, что

$$
\begin{aligned}
y_{t} \bar{y}_{t+1} & =\left(x_{t+1}+x_{t} x_{t+2} \bar{x}_{t+3}\right)\left(\bar{x}_{t+2}+x_{t+1} x_{t+3} \bar{x}_{t+4}\right)= \\
& =x_{t+1}\left(\bar{x}_{t+2}+x_{t+1} x_{t+3} \bar{x}_{t+4}\right)=x_{t+1} \bar{y}_{t+1}, \\
y_{t-2} y_{t} \bar{y}_{t+1} & =\left(x_{t-1}+x_{t-2} x_{t} \bar{x}_{t+1}\right) x_{t+1} \bar{y}_{t+1}=x_{t-1} x_{t+1} \bar{y}_{t+1} .
\end{aligned}
$$

Проведя аналогичные преобразования для остальных сомножителей, получим:

$$
y_{0} \cdot \ldots \cdot y_{t-2} y_{t} \bar{y}_{t+1}=x_{1} x_{3} \cdot \ldots \cdot x_{t-1} x_{t+1} \bar{y}_{t+1} .
$$

Так как $y_{n-2}=x_{n-1}+x_{n-2} x_{0} \bar{x}_{1}$,

$$
\begin{aligned}
h(\boldsymbol{y})= & y_{n-1}+\left(x_{n-1}+x_{n-2} x_{0} \bar{x}_{1}\right)\left[\sum_{t=0, t \text {-четно }}^{n-5} x_{1} x_{3} \cdot \ldots \cdot x_{t-1} x_{t+1} \bar{y}_{t+1}\right]= \\
= & y_{n-1}+x_{n-1}\left[\sum_{t=0, t \text {-четно }}^{n-5} x_{1} x_{3} \cdot \ldots \cdot x_{t-1} x_{t+1}\left(\bar{x}_{t+2}+x_{t+1} x_{t+3} \bar{x}_{t+4}\right)\right]= \\
= & y_{n-1}+x_{n-1} \cdot\left[\sum_{t=0, t \text {-четно }}^{n-5} x_{1} x_{3} \cdot \ldots \cdot x_{t-1} x_{t+1} \bar{x}_{t+2}+x_{1} x_{3} \cdot \ldots \times\right. \\
& \left.\left.\times x_{t-1} x_{t+1} x_{t+3} \bar{x}_{t+4}\right)\right]= \\
= & \left.y_{n-1}+x_{n-1} \cdot\left[x_{1} \bar{x}_{2}+x_{1} x_{3} \cdot \ldots \cdot x_{t-1} x_{t+1} x_{t+3} \bar{x}_{n-1}\right)\right]= \\
= & x_{n}+x_{n-1} x_{n+1} \bar{x}_{n+2}+x_{1} \bar{x}_{2} x_{n-1}=x_{0}+x_{n-1} x_{1} \bar{x}_{2}+x_{1} \bar{x}_{2} x_{n-1}=x_{0} .
\end{aligned}
$$

3) Если $n$ кратно 3, то группа $\langle\sigma\rangle$ имеет две орбиты мощности 3 : $\{(0,0,1,0,0,1 \ldots 0,0,1),(0,1,0,0,1,0 \ldots 0,1,0),(1,0,0,1,0,0 \ldots 1,0,0)\}$ и $\{(1,1,0,1,1,0 \ldots 1,1,0),(1,0,1,1,0,1 \ldots 1,0,1),(0,1,1,0,1,1 \ldots 0,1,1)\}$.

На векторах этих орбит функции $x_{0}+x_{1} x_{2} \bar{x}_{3}$ и $x_{0}+\bar{x}_{1} x_{2} x_{3}$ принимают постоянное значение и не порождают регулярных систем $\langle\sigma\rangle$-однотипных функций при кратных 3 значениях $n$.

Пусть $n \geq 5, n$ не делится на $3, f(\boldsymbol{x})=x_{0}+x_{1} x_{2} \bar{x}_{3}$. Тогда

$$
y_{i}=x_{i}+x_{i+1} x_{i+2} \bar{x}_{i+3}, \quad i \in \overline{0, n-1} \text {. }
$$

Покажем, что

$$
h(\boldsymbol{y})=y_{0}+\sum_{t=1}^{\left[\frac{n}{3}\right]}\left[\prod_{j=1}^{t} y_{3 j-2} y_{3 j-1}\right] \bar{y}_{3 t} .
$$


Для фиксированного $t \in \overline{1,\left[\frac{n}{3}\right]}$ в слагаемом

$$
\left[\prod_{j=1}^{t} y_{3 j-2} y_{3 j-1}\right] \bar{y}_{3 t}=y_{1} y_{2} y_{4} y_{5} y_{7} y_{8} \cdot \ldots \cdot y_{3 t-4} y_{3 t-2} y_{3 t-1} \bar{y}_{3 t}
$$

отсутствуют сомножители с индексами, кратными 3, кроме последнего с индексом $3 t$. Заметим, что

$$
\begin{aligned}
& y_{3 t-1} \bar{y}_{3 t}=\left(x_{3 t-1}+x_{3 t} x_{3 t+1} \bar{x}_{3 t+2}\right)\left(\bar{x}_{3 t}+x_{3 t+1} x_{3 t+2} \bar{x}_{3 t+3}\right)= \\
& =x_{3 t-1}\left(\bar{x}_{3 t}+x_{3 t+1} x_{3 t+2} \bar{x}_{3 t+3}\right)=x_{3 t-1} \bar{y}_{3 t} \text {. } \\
& y_{3 t-2} y_{3 t-1} \bar{y}_{3 t}=\left(x_{3 t-2}+x_{3 t-1} x_{3 t} \bar{x}_{3 t+1}\right) x_{3 t-1}\left(\bar{x}_{3 t}+x_{3 t+1} x_{3 t+2} \bar{x}_{3 t+3}\right)= \\
& =x_{3 t-2} x_{3 t-1}\left(\bar{x}_{3 t}+x_{3 t+1} x_{3 t+2} \bar{x}_{3 t+3}\right)=x_{3 t-2} x_{3 t-1} \bar{y}_{3 t} \text {. } \\
& y_{3 t-4} y_{3 t-2} y_{3 t-1} \bar{y}_{3 t}=\left(x_{3 t-4}+x_{3 t-3} x_{3 t-2} \bar{x}_{3 t-1}\right) x_{3 t-2} \times \\
& \times x_{3 t-1}\left(\bar{x}_{3 t}+x_{3 t+1} x_{3 t+2} \bar{x}_{3 t+3}\right)= \\
& =x_{3 t-4} x_{3 t-2} x_{3 t-1}\left(\bar{x}_{3 t}+x_{3 t+1} x_{3 t+2} \bar{x}_{3 t+3}\right)=x_{3 t-4} x_{3 t-2} x_{3 t-1} \bar{y}_{3 t} \text {. }
\end{aligned}
$$

Продолжая аналогично и используя выражение $y_{i}, i \in\{0, \ldots, n-1\}$, через $x_{i}, i \in\{0, \ldots, n-1\}$, а также учитывая, что в рассматриваемом слагаемом отсутствует каждый третий сомножитель, получим:

$y_{1} y_{2} y_{4} y_{5} y_{7} y_{8} \cdot \ldots \cdot y_{3 t-4} y_{3 t-2} y_{3 t-1} \bar{y}_{3 t}=x_{1} x_{2} x_{4} x_{5} x_{7} x_{8} \cdot \ldots \cdot x_{3 t-4} x_{3 t-2} x_{3 t-1} \bar{y}_{3 t}$.

Отсюда следует, что для соседних слагаемых с номерами $t$ и $t+1$

$$
\begin{gathered}
y_{1} y_{2} y_{4} \cdot \ldots \cdot y_{3 t-4} y_{3 t-2} y_{3 t-1} \bar{y}_{3 t}+y_{1} y_{2} y_{4} \cdot \ldots \cdot y_{3 t-4} y_{3 t-2} y_{3 t-1} y_{3 t+1} y_{3 t+2} \bar{y}_{3 t+3}= \\
=x_{1} x_{2} x_{4} \cdot \ldots \cdot x_{3 t-4} x_{3 t-2} x_{3 t-1}\left[\bar{y}_{3 t}+x_{3 t+1} x_{3 t+2} \bar{y}_{3 t+3}\right]= \\
=x_{1} x_{2} x_{4} \cdot \ldots \cdot x_{3 t-4} x_{3 t-2} x_{3 t-1} \times \\
\times\left[\bar{x}_{3 t}+x_{3 t+1} x_{3 t+2} \bar{x}_{3 t+3}+x_{3 t+1} x_{3 t+2}\left(\bar{x}_{3 t+3}+x_{3 t+4} x_{3 t+5} \bar{x}_{3 t+6}\right)\right]= \\
=x_{1} x_{2} x_{4} \cdot \ldots \cdot x_{3 t-4} x_{3 t-2} x_{3 t-1}\left[\bar{x}_{3 t}+x_{3 t+1} x_{3 t+2} x_{3 t+4} x_{3 t+5} \bar{x}_{3 t+6}\right] .
\end{gathered}
$$

Поэтому в сумме $\sum_{t=1}^{\left[\frac{n}{3}\right]}\left[\prod_{j=1}^{t} y_{3 j-2} y_{3 j-1}\right] \bar{y}_{3 t}$ после выражения переменных $y_{i}$, $i \in\{0, \ldots, n-1\}$, через $x_{i}, i \in\{0, \ldots, n-1\}$, останется только два ненулевых слагаемых

$$
x_{1} x_{2} \bar{x}_{3} \quad \text { и } \quad x_{1} x_{2} x_{4} \cdot \ldots \cdot x_{3\left[\frac{n}{3}\right]-2} x_{3\left[\frac{n}{3}\right]-1} x_{3\left[\frac{n}{3}\right]+1} x_{3\left[\frac{n}{3}\right]+2} \bar{x}_{3\left[\frac{n}{3}\right]+3} \cdot
$$

После приведения индексов по модулю $n$ конъюнкция

$$
x_{1} x_{2} x_{4} \cdot \ldots \cdot x_{3\left[\frac{n}{3}\right]-2} x_{3\left[\frac{n}{3}\right]-1} x_{3\left[\frac{n}{3}\right]+1} x_{3\left[\frac{n}{3}\right]+2} \bar{x}_{3\left[\frac{n}{3}\right]+3}
$$


будет содержать все переменные с индексами от 0 до $n-1$, кроме переменных, индексы которых кратны 3. Покажем, что конъюнкция (23) равна 0. Для этого покажем, что среди переменных, входящих без инверсии, есть переменная с индексом $\left(3\left[\frac{n}{3}\right]+3\right) \bmod n$. Поскольку $n$ не кратно 3 , для некоторого $k \in \mathbb{N}$ и $r \in\{1,2\}$ выполняется равенство $n=3 k+r$. Тогда

$$
3\left[\frac{n}{3}\right]+3=3\left[\frac{3 k+r}{3}\right]+3=3 k+3=3 k+r+(3-r) .
$$

Значит, $3\left[\frac{n}{3}\right]+3 \equiv(3-r) \bmod n$. Поскольку $r \in\{1,2\}$, после приведения индексов переменных по модулю $n$ конъюнкция (23) будет содержать переменные $x_{3-r}$ и $\bar{x}_{3-r}$.

В итоге получим, что

$h(\boldsymbol{y})=y_{0}+\sum_{t=1}^{\left[\frac{n}{3}\right]}\left[\prod_{j=1}^{t} y_{3 j-2} y_{3 j-1}\right] \bar{y}_{3 t}=y_{0}+x_{1} x_{2} \bar{x}_{3}=x_{0}+x_{1} x_{2} \bar{x}_{3}+x_{1} x_{2} \bar{x}_{3}=x_{0}$.

Пусть $n \geq 5, n$ не делится на $3, f(\boldsymbol{x})=x_{0}+\bar{x}_{1} x_{2} x_{3}$.

Имеем: $y_{i}=x_{i}+\bar{x}_{i+1} x_{i+2} x_{i+3}, i \in\{0, \ldots, n-1\}$. Покажем, что

$$
h(\boldsymbol{y})=y_{0}+\sum_{t=1}^{\left[\frac{n}{3}\right]}\left[\prod_{j=1}^{t} \bar{y}_{3 j-2} y_{3 j-1}\right] y_{3 t} \text {. }
$$

Для фиксированного $t \in\left\{1, \ldots,\left[\frac{n}{3}\right]\right\}$ рассмотрим слагаемое

$$
\left[\prod_{j=1}^{t} \bar{y}_{3 j-2} y_{3 j-1}\right] y_{3 t}=\bar{y}_{1} y_{2} \bar{y}_{4} y_{5} \bar{y}_{7} y_{8} \cdot \ldots \cdot \bar{y}_{3 t-2} y_{3 t-1} y_{3 t} .
$$

Как и в предыдущем случае, в данном слагаемом отсутствуют сомножители с индексами кратными 3 , кроме последнего с индексом $3 t$. Заметим, что

$$
\begin{gathered}
y_{3 t-1} y_{3 t}=\left(x_{3 t-1}+\bar{x}_{3 t} x_{3 t+1} x_{3 t+2}\right)\left(x_{3 t}+\bar{x}_{3 t+1} x_{3 t+2} x_{3 t+3}\right)= \\
=x_{3 t-1}\left(x_{3 t}+\bar{x}_{3 t+1} x_{3 t+2} x_{3 t+3}\right)=x_{3 t-1} y_{3 t} \\
\bar{y}_{3 t-2} y_{3 t-1} y_{3 t}=\left(\bar{x}_{3 t-2}+\bar{x}_{3 t-1} x_{3 t} x_{3 t+1}\right) x_{3 t-1}\left(x_{3 t}+\bar{x}_{3 t+1} x_{3 t+2} x_{3 t+3}\right)= \\
=\bar{x}_{3 t-2} x_{3 t-1}\left(x_{3 t}+\bar{x}_{3 t+1} x_{3 t+2} x_{3 t+3}\right)=\bar{x}_{3 t-2} x_{3 t-1} y_{3 t} . \\
y_{3 t-4} \bar{y}_{3 t-2} y_{3 t-1} y_{3 t}=\left(x_{3 t-4}+\bar{x}_{3 t-3} x_{3 t-2} x_{3 t-1}\right) \bar{x}_{3 t-2} \times \\
\times x_{3 t-1}\left(x_{3 t}+\bar{x}_{3 t+1} x_{3 t+2} x_{3 t+3}\right)= \\
=x_{3 t-4} \bar{x}_{3 t-2} x_{3 t-1}\left(x_{3 t}+\bar{x}_{3 t+1} x_{3 t+2} x_{3 t+3}\right)=x_{3 t-4} \bar{x}_{3 t-2} x_{3 t-1} y_{3 t} .
\end{gathered}
$$


Продолжая аналогично и используя выражение $y_{i}, i \in\{0, \ldots, n-1\}$, через $x_{i}, i \in\{0, \ldots, n-1\}$, а также учитывая, что в рассматриваемом слагаемом отсутствует каждый третий сомножитель, получим:

$$
\bar{y}_{1} y_{2} \bar{y}_{4} y_{5} \bar{y}_{7} y_{8} \cdot \ldots \cdot \bar{y}_{3 t-2} y_{3 t-1} y_{3 t}=\bar{x}_{1} x_{2} \bar{x}_{4} x_{5} \bar{x}_{7} x_{8} \cdot \ldots \cdot \bar{x}_{3 t-2} x_{3 t-1} y_{3 t} .
$$

Для соседних слагаемых с номерами $t$ и $t+1$ получаем соотношения

$$
\begin{gathered}
\bar{y}_{1} y_{2} \bar{y}_{4} y_{5} \bar{y}_{7} y_{8} \cdot \ldots \cdot \bar{y}_{3 t-2} y_{3 t-1} y_{3 t}+ \\
+\bar{y}_{1} y_{2} \bar{y}_{4} y_{5} \bar{y}_{7} y_{8} \cdot \ldots \cdot \bar{y}_{3 t-2} y_{3 t-1} \bar{y}_{3 t+1} y_{3 t+2} y_{3 t+3}= \\
=\bar{x}_{1} x_{2} \bar{x}_{4} \cdot \ldots \cdot x_{3 t-4} \bar{x}_{3 t-2} x_{3 t-1}\left[y_{3 t}+\bar{x}_{3 t+1} x_{3 t+2} y_{3 t+3}\right]= \\
=\bar{x}_{1} x_{2} \bar{x}_{4} \cdot \ldots \cdot x_{3 t-4} \bar{x}_{3 t-2} x_{3 t-1} \times \\
\times\left[x_{3 t}+\bar{x}_{3 t+1} x_{3 t+2} x_{3 t+3}+\bar{x}_{3 t+1} x_{3 t+2}\left(x_{3 t+3}+\bar{x}_{3 t+4} x_{3 t+5} x_{3 t+6}\right)\right]= \\
=\bar{x}_{1} x_{2} \bar{x}_{4} \cdot \ldots \cdot x_{3 t-4} \bar{x}_{3 t-2} x_{3 t-1}\left[x_{3 t}+\bar{x}_{3 t+1} x_{3 t+2} \bar{x}_{3 t+4} x_{3 t+5} x_{3 t+6}\right] .
\end{gathered}
$$

Поэтому в сумме $\sum_{t=1}^{\left[\frac{n}{3}\right]}\left[\prod_{j=1}^{t} \bar{y}_{3 j-2} y_{3 j-1}\right] y_{3 t}$ после выражения переменных $y_{i}$, $i \in\{0, \ldots, n-1\}$, через $x_{i}, i \in\{0, \ldots, n-1\}$, останется только два ненулевых слагаемых

$$
\bar{x}_{1} x_{2} x_{3} \quad \text { и } \quad \bar{x}_{1} x_{2} \bar{x}_{4} \cdot \ldots \cdot \bar{x}_{3\left[\frac{n}{3}\right]-2} x_{3\left[\frac{n}{3}\right]-1} \bar{x}_{3\left[\frac{n}{3}\right]+1} x_{3\left[\frac{n}{3}\right]+2} x_{3\left[\frac{n}{3}\right]+3} .
$$

После приведения индексов по модулю $n$ коньюнкция

$$
\bar{x}_{1} x_{2} \bar{x}_{4} \cdot \ldots \cdot \bar{x}_{3\left[\frac{n}{3}\right]-2} x_{3\left[\frac{n}{3}\right]-1} \bar{x}_{3\left[\frac{n}{3}\right]+1} x_{3\left[\frac{n}{3}\right]+2} x_{3\left[\frac{n}{3}\right]+3}
$$

будет содержать все переменные с индексами от 0 до $n-1$, кроме переменных, индексы которых кратны 3. Аналогично предыдущему случаю покажем, что конъюнкция (24) равна 0.

Если $n=3 k+1, k \in \mathbb{N}$, то

$3\left[\frac{n}{3}\right]+2=3\left[\frac{3 k+1}{3}\right]+2=3 k+2=3 k+1+1=1 \quad(\bmod n)$.

Если $n=3 k+2, k \in \mathbb{N}$, то

$3\left[\frac{n}{3}\right]+3=3\left[\frac{3 k+2}{3}\right]+3=3 k+3=3 k+2+1=1 \quad(\bmod n)$.

Таким образом, если $n$ не кратно 3, то конъюнкция (24) содержит переменные $\bar{x}_{1}$ и $x_{1}$. Поэтому

$h(\boldsymbol{y})=y_{0}+\sum_{t=1}^{\left[\frac{n}{3}\right]}\left[\prod_{j=1}^{t} \bar{y}_{3 j-2} y_{3 j-1}\right] y_{3 t}=y_{0}+\bar{x}_{1} x_{2} x_{3}=x_{0}+\bar{x}_{1} x_{2} x_{3}+\bar{x}_{1} x_{2} x_{3}=x_{0}$. 
Заметим, что

$$
\left(x_{0}, \ldots, x_{n-1}\right)^{\omega \sigma^{4}}=\left(x_{3}, x_{2}, x_{1}, x_{0}, x_{n-1}, \ldots, x_{4}\right) .
$$

Тогда:

1) $f^{\sigma^{-4} \omega}=x_{2}+x_{0} \bar{x}_{1} x_{3}$ для $f=x_{1}+x_{0} \bar{x}_{2} x_{3}$;

2) $f^{\sigma^{-4} \omega}=x_{2}+\bar{x}_{0} x_{1} x_{3}$ для $f=x_{1}+x_{0} x_{2} \bar{x}_{3}$;

3) (a) $f^{\sigma^{-4} \omega}=x_{3}+\bar{x}_{0} x_{1} x_{2}$ для $f=x_{0}+x_{1} x_{2} \bar{x}_{3}$,

(b) $f^{\sigma^{-4} \omega}=x_{3}+x_{0} x_{1} \bar{x}_{2}$ для $f=x_{0}+\bar{x}_{1} x_{2} x_{3}$.

Воспользовавшись леммой 3, завершаем доказательство теоремы 4.

Новые классы функций третьей степени, существенно зависящих от первых четырех переменных и порождающих регулярные системы $\langle\sigma\rangle$-однотипных функций, могут быть построены с использованием следующего очевидного утверждения.

Утверждение 1. Если система $\mathcal{C}(f ; \sigma)$ регулярна, то системь

$$
\mathcal{C}(\bar{f} ; \sigma) \quad \text { u } \quad \mathcal{C}\left(f^{*} ; \sigma\right),
$$

где $f^{*}\left(x_{0}, \ldots, x_{n-1}\right)=f\left(\bar{x}_{0}, \ldots, \bar{x}_{n-1}\right)$, также являются регулярными.

Заметим, что построенные классы функций имеют индекс $t=4$. Действительно, как нетрудно видеть, все функции однотипны, а значит, имеют одинаковый индекс. Функция

$$
f=x_{0}+x_{1} x_{2} \bar{x}_{3}
$$

имеет кратчайшую ДНФ вида

$$
x_{0} x_{3} \vee x_{0} \bar{x}_{1} \bar{x}_{3} \vee x_{0} \bar{x}_{2} \bar{x}_{3} \vee \bar{x}_{0} x_{1} x_{2} \bar{x}_{3} .
$$

\section{Список литературы}

1. Blake A. Canonical Expression in Boolean Algebra. - Chicago, 1937.

2. Журавлёв Ю.И. Алгоритмы построения минимальных д. н. ф. для функций алгебры логики. - В сб.: Дискретная математика и математические вопросы кибернетики. - М.: Наука, 1974, с. 67-82.

3. Журавлёв Ю. И. Об алгоритмах упрощения дизъюнктивных нормальных форм. Докл. АН СССР, 1960, т. 132, № 2, с. 260-263. 
4. Закревский А. Д. Логический синтез каскадных схем. - М.: Наука, 1981.

5. Quine $W . V$. The problem of simplifying truth function. - Amer. Math. Monthly, 1952, v. 59, p. 521-531.

6. Mc Cluskey E. J., Jr. Minimization of boolean functions. - Bell Syst. Techn. J., 1956, v. 35, № 6, p. 1417-1444.

7. Нигматуллин Р. Г. Сложность булевых функций. - М.: Наука, 1991.

8. Никонов В. Г. Покрытия булевых графов. - Дискрет. матем., 1994, т. 6, в. 4.

9. Никонов В. Г. Классификация минимальных базисных представлений всех булевых функций от четырех переменных. - Обозрение прикл. промышл. матем., 1994 , т. 1 , в. 3 , с. $458-545$.

10. Никонов В.Г. Пороговые представления булевых функций. - Обозрение прикл. промышл. матем. Сер. дискрет. матем., 1994, т. 1, в. 3, с. 402-458.

11. Никонов В.Г. Конечность некоторых классов булевых функций с ограниченным числом элементарных конъюнкций в ДНФ. - Вестник РУДН, Сер. Прикл. и комп. математика, 2003, т. 2, № 1, с. 68-78.

12. Никонов В. Г. О существовании минимальной, но не кратчайшей ортогональной дизъюнктивной нормальной формы. - В сб.: Труды по дискретной математике. Т. 10. - М.: ФИЗМАТЛИТ, 2007, с. 188-201.

13. Никонов В. Г., Саранцев А. В. Методы компактной реализации биективных отображений, заданных регулярными системами однотипных булевых функций. Вестник РУДН, Сер. Прикл. и комп. математика, 2003, т. 2, № 1, с. 84-95.

14. Саранцев $A$. B. Построение регулярных систем однотипных двоичных функций с использованием регистра сдвига. - Вестник МГУЛ, 2004, № 1 (32), с. 164-169.

15. Саранцев А. В. Регулярные системы однотипных двоичных функций степени 3 , построенные с помощью циклического сдвига. - Обозрение прикл. и промышл. матем., 2007, т. 14 , в. 1, с. 147-148. 